\title{
Reduction of Nonlinear Effects in Magnetohydrodynamic and Hall Magnetohydrodynamic Turbulence*)
}

\author{
Keisuke ARAKI and Hideaki MIURA ${ }^{1)}$ \\ Okayama University of Science, Okayama 700-0005, Japan \\ ${ }^{1)}$ National Institute for Fusion Science, Toki 509-5292, Japan
}

(Received 22 November 2012 / Accepted 21 June 2013)

\begin{abstract}
We analyzed energy transfer processes induced by triad mode interactions in homogeneous, isotropic turbulence in magnetohydrodynamic (MHD) and Hall magnetohydrodynamic (HMHD) media. In particular, we analyzed the Fourier spectra of the energy transfers using geometric-series shell-partitioning methods, analogous to dyadic wavelet analysis. Snapshot datasets were collected once the MHD and HMHD turbulences had sufficiently developed. Graphs of all energy spectra were well collapsed after the normalization using the dissipation rates and the diffusion coefficients, i.e. they showed good self-similarity. Despite such self-similarity of energy spectra, the transfer due to mode interactions between the fluid advection and Hall term was reduced over time, while those due to the Lorentz force and induction remained rather stationary in regions of higher wave number.

(C) 2013 The Japan Society of Plasma Science and Nuclear Fusion Research
\end{abstract}

Keywords: Hall magnetohydrodynamics, turbulence, energy transfer, wavelet analysis

DOI: $10.1585 /$ pfr.8.2401137

\section{Introduction}

Magnetohydrodynamics (MHD) turbulence is relevant to a wide range of research topics, including astrophysics and fusion plasma studies. In recent years, extensions of MHD equations to two-fluid effects have attracted much attention [1].

A simple fluid model incorporating a two-fluid effect is the Hall MHD (HMHD) system. The MHD and HMHD systems differ significantly in their energy dissipation tendencies, structure formation processes, and profiles of the generated coherent structures [2]. It appears that the Hall term maintains large-scale spatial structures in fully developed turbulence, which is important for plasma confinement.

We also compared the wavelet-scale spectra of the energy exchange generated by the induction term in a snapshot dataset. Local energy transfer dominated the exchange, and energy transfer to small scales was observed in both cases [3]. By analyzing several serial snapshot datasets, we also found that the Hall term induces nonlinear energy transfer over large scales, a phenomenon that is intrinsic to HMHD dynamics [4]. In particular, nonlocal interactions between the modes of a magnetic field are crucial to back scattering phenomena.

Despite these advances, how the nonlocal interaction of the Hall term affects the energy transfer processes in HMHD and MHD systems is yet to be clarified. In the present study, we investigate the temporal dynamics of the energy transfer processes in HMHD and compare them

author'se-mail: araki@are.ous.ac.jp

*) This article is based on the presentation at the 22nd International Toki Conference (ITC22). with those of MHD over longer time intervals than in our previous studies.

\section{Basic Equations and Numerical Simulation}

To exclude forcing influences, we analyze a freely decaying, homogeneous, isotropic turbulence in an incompressible HMHD system. The incompressible HMHD equations are given by

$$
\begin{aligned}
& \frac{\partial \boldsymbol{u}}{\partial t}+(\boldsymbol{u} \cdot \nabla) \boldsymbol{u}=-\nabla P+\boldsymbol{j} \times \boldsymbol{b}+v \nabla^{2} \boldsymbol{u}, \\
& \frac{\partial \boldsymbol{b}}{\partial t}=\nabla \times((\boldsymbol{u}-\epsilon \boldsymbol{j}) \times \boldsymbol{b})+\eta \nabla^{2} \boldsymbol{b},
\end{aligned}
$$

where the bulk velocity field $\boldsymbol{u}$ satisfies $\nabla \cdot \boldsymbol{u}=0, \boldsymbol{b}$ is the magnetic field, $\boldsymbol{j}:=\nabla \times \boldsymbol{b}$ is the current density field, $P$ is the total pressure, $v$ is the kinematic viscosity, $\eta$ is the resistivity, and $\epsilon$ is parameter specifying the relative strength of the Hall term. The numerical schemes are detailed in [2].

The parameters are set to $v=\eta=1 \times 10^{-3}$ and $\epsilon=0.05$. In a comparison simulation, we set $\epsilon=0 l \mathrm{Cal}-$ culations were performed on $N^{3}=512^{3}$ grid points. We adopted the pseudospectral method with a $2 / 3$ dealiasing rule $\left(k_{\max }=170\right)$. To elucidate the effects of the Hall term on the solutions, both simulations were conducted under the same initial conditions.

\section{Analysis Method}

Since a freely decaying turbulence was imposed, the amplitudes and characteristic scales of turbulent velocity and magnetic fields gradually altered over time. To quan- 


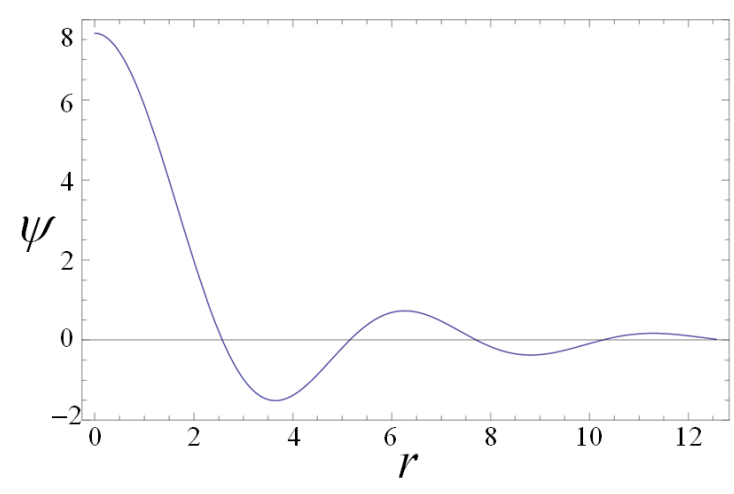

Fig. 1 Profile of filtering function $\psi(r)$.

titatively capture the universal features, we must normalize the datasets and apply an appropriate filtering method. In the present study, we quantitatively compared the interscale energy transfer budget due to triad mode interactions between the snapshots collected at different times using a combination of analysis techniques.

First, each set of field data was normalized by the resistivity coefficient $\eta$ and the dissipation rate of the magnetic energy $\epsilon_{B}(t)$. As shown in [4], the amplitudes of the energy spectra and the energy transfer spectra are of comparable order among the different time snapshots.

To evaluate the shell-averaged energy budget imposed by mode interactions, each field was decomposed into band-pass-filtered components as

$$
f(\vec{x}, t)=\sum f_{j}(\vec{x}, t),
$$

where $f$ incorporates the velocity and magnetic fields and $j$ is the scale index of the filtered field. Each filtered component is the sum of the Fourier modes on a spherical shell in the wavenumber space:

$$
f_{j}(\vec{x}, t):=\sum_{|\vec{k}|=k_{\eta} / \sqrt{2^{j}}}^{k_{\eta} / \sqrt{2^{j-1}}} \hat{f}(\vec{k}, t) \exp (2 \pi i \vec{k} \cdot \vec{x} / L),
$$

where $k_{\eta}(t):=\sqrt[4]{\epsilon_{B}(t) / \eta^{3}}$ is the characteristic wavenumber of the dissipation range and $L$ is the system size. The characteristic oscillation wave length $\lambda_{j}$ and the envelope scale of $\boldsymbol{f}_{j}$ (also known as the window width of the band pass filter) $\Delta_{j}$ obey the following scaling relations

$$
\lambda_{j} \propto \sqrt{2^{j}}, \quad \Delta_{j} \propto \sqrt{2^{j}} .
$$

Alternatively, geometric series shell partitioning in Fourier space is achieved by convolution with a mollifier:

$$
\boldsymbol{f}_{j}(\vec{x}, t)=\left(\frac{k_{\eta}}{\sqrt{2}}\right)^{3} \int \psi\left(\frac{k_{\eta}|\vec{y}|}{\sqrt{2^{j}}}\right) \boldsymbol{f}(\vec{x}-\vec{y}, t) \mathrm{d}^{3} \vec{y},
$$

where the mollifier $\psi$ is given by

$$
\psi(r)=\frac{4 \pi}{r^{3}}[r \cos r-\sin r-\sqrt{2} r \cos \sqrt{2} r+\sin \sqrt{2} r]
$$

(see Fig. 1). We remark that this decomposition is analo- gous to "dyadic wavelet" analysis [5].

In the present study, we analyzed the energy budget in the band-pass-filtered representation. The energy budget of MHD and HMHD turbulence was analyzed as described in [3], except that the wavelet scale decomposition was replaced with the $\sqrt{2}$-adic scale decomposition given by Eq. (3).

$$
\begin{gathered}
\frac{\mathrm{d}}{\mathrm{d} t} E_{j}^{(u)}=\sum_{k, m}\left\langle\boldsymbol{u}_{j}\left|\boldsymbol{u}_{m}\right| \boldsymbol{u}_{k}\right\rangle_{a d v}+\sum_{k, m}\left\langle\boldsymbol{u}_{j}\left|\boldsymbol{b}_{m}\right| \boldsymbol{b}_{k}\right\rangle_{L o r}+D_{j}, \\
\frac{\mathrm{d}}{\mathrm{d} t} E_{k}^{(b)}=\sum_{j, m}\left\langle\boldsymbol{b}_{k}\left|\boldsymbol{b}_{m}\right| \boldsymbol{u}_{j}\right\rangle_{I n d}+\sum_{j, m}\left\langle\boldsymbol{b}_{k}\left|\boldsymbol{b}_{m}\right| \boldsymbol{b}_{j}\right\rangle_{\text {Hall }}+R_{k},
\end{gathered}
$$

where

$$
\begin{aligned}
& E_{j}^{(u)}:=\frac{1}{2} \int\left|\boldsymbol{u}_{j}\right|^{2} \mathrm{~d}^{3} \vec{x}, D_{j}:=-v \int\left|\nabla \times \boldsymbol{u}_{j}\right|^{2} \mathrm{~d}^{3} \vec{x} \\
& E_{k}^{(b)}:=\frac{1}{2} \int\left|\boldsymbol{b}_{k}\right|^{2} \mathrm{~d}^{3} \vec{x}, R_{k}:=-\eta \int\left|\nabla \times \boldsymbol{b}_{k}\right|^{2} \mathrm{~d}^{3} \vec{x} \\
& \left\langle\boldsymbol{u}_{j}\left|\boldsymbol{u}_{m}\right| \boldsymbol{u}_{k}\right\rangle_{a d v}:=-\int\left(\left(\boldsymbol{u}_{m} \cdot \nabla\right) \boldsymbol{u}_{k}\right) \cdot \boldsymbol{u}_{j} \mathrm{~d}^{3} \vec{x} \\
& \left\langle\boldsymbol{u}_{j}\left|\boldsymbol{b}_{m}\right| \boldsymbol{b}_{k}\right\rangle_{L o r}:=\int\left(\boldsymbol{j}_{k} \times \boldsymbol{b}_{m}\right) \cdot \boldsymbol{u}_{j} \mathrm{~d}^{3} \vec{x} \\
& \left\langle\boldsymbol{b}_{k}\left|\boldsymbol{b}_{m}\right| \boldsymbol{u}_{j}\right\rangle_{\text {Ind }}:=\int\left(\nabla \times\left(\boldsymbol{u}_{j} \times \boldsymbol{b}_{m}\right)\right) \cdot \boldsymbol{b}_{k} \mathrm{~d}^{3} \vec{x} \\
& \left\langle\boldsymbol{b}_{k}\left|\boldsymbol{b}_{m}\right| \boldsymbol{b}_{j}\right\rangle_{\text {Hall }}:=-\epsilon \int\left(\nabla \times\left(j_{k} \times \boldsymbol{b}_{m}\right)\right) \cdot \boldsymbol{b}_{j} \mathrm{~d}^{3} \vec{x}
\end{aligned}
$$

The integrant in the RHS of Eqs. (8)-(11) were carefully chosen to satisfy covariance under arbitrary changes of the coordinate system (see [6]) and the following reciprocal relations:

$$
\begin{aligned}
& \left\langle\boldsymbol{u}_{j}\left|\boldsymbol{u}_{m}\right| \boldsymbol{u}_{k}\right\rangle_{\text {adv }}=-\left\langle\boldsymbol{u}_{k}\left|\boldsymbol{u}_{m}\right| \boldsymbol{u}_{j}\right\rangle_{\text {adv }}, \\
& \left\langle\boldsymbol{u}_{j}\left|\boldsymbol{b}_{m}\right| \boldsymbol{b}_{k}\right\rangle_{\text {Lor }}=-\left\langle\boldsymbol{b}_{k}\left|\boldsymbol{b}_{m}\right| \boldsymbol{u}_{j}\right\rangle_{\text {Ind }}, \\
& \left\langle\boldsymbol{b}_{k}\left|\boldsymbol{b}_{m}\right| \boldsymbol{b}_{j}\right\rangle_{\text {Hall }}=-\left\langle\boldsymbol{b}_{j}\left|\boldsymbol{b}_{m}\right| \boldsymbol{b}_{k}\right\rangle_{\text {Hall }} .
\end{aligned}
$$

\section{Numerical Results}

We analyzed snapshot datasets collected between $t=$ 1.0 and 5.0 with time increment 0.5 . The selected time interval is sufficiently beyond the time of maximum vorticity and current density fields. As shown in Fig. 2, the temporal development of the Taylor microscale Reynolds number and its magnetic counterpart, defined by

$$
\operatorname{Re}:=\sqrt{\frac{10}{3 v \epsilon}} E^{(u)}, \operatorname{Rem}:=\sqrt{\frac{10}{3 \eta \epsilon_{B}}} E^{(b)},
$$

become settled at $t \gtrsim 1.0$. The magnetic Reynolds numbers are approximately twice the Reynolds number. This implies that, in the present solutions, the magnetic field is more energetic than the fluid motion, independent of 


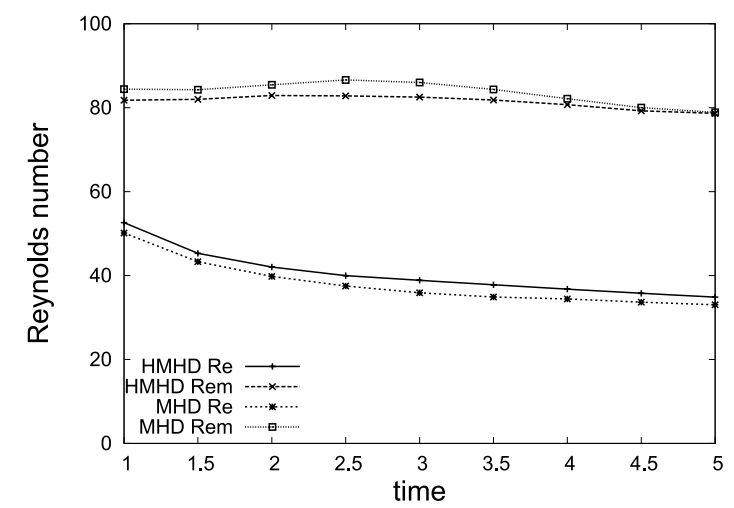

Fig. 2 Time development of the Taylor microscale Reynolds number and its magnetic counterpart.

the Hall term effect. The characteristics of these snapshot datasets probably reflect self-similar features of spontaneously generated MHD and HMHD turbulence.

Throughout the analyzed time period, each functional form of the normalized Fourier spectra collapsed into a single form, indicating that the turbulent MHD and HMHD fields both exist in self-similar states (see Fig. 3).

Figures 4 and 5 plot the quadratic terms of the shellaveraged energy transfers. As expected, normalization renders the amplitudes of the energy transfer functions comparable between different time snapshots in both HMHD and MHD. Thus, the key physical parameters are the dissipation coefficient and the energy dissipation rate. In other words, the principal dynamic features are dominated by those of the dissipation range. Here, the physical quantities are normalized by the resistivity coefficient and dissipation rate of magnetic energy. However, normalization by the viscosity coefficient and dissipation rate of kinetic energy yields qualitatively similar results.

Moreover, the peaks of the transfer functions generated by the Lorentz force and magnetic induction are closely spaced in the HMHD and MHD solutions. Peak values of transfer by the Lorentz force are close to each other in both systems. The same tendency is seen for the magnetic induction effect.

In both systems, the energy is overall transferred to smaller scales, while weak inverse transfer to larger scales is observed in the fluid advection effect. All the analyzed snapshots show inverse energy transfer induced by the Hall term.

In the HMHD simulations (see Fig.4), the energy transfer spectra of the Lorentz force and magnetic induction terms show similar form for $k \gtrsim 0.1 k_{\eta}(t)$. This suggests that over dissipation range scales, the statistical features of the mode interaction between the normalized magnetic and velocity fields rapidly converge to the equilibrium state.

On the contrary, although the normalized spectra are of comparable amplitude, the energy transfers induced by
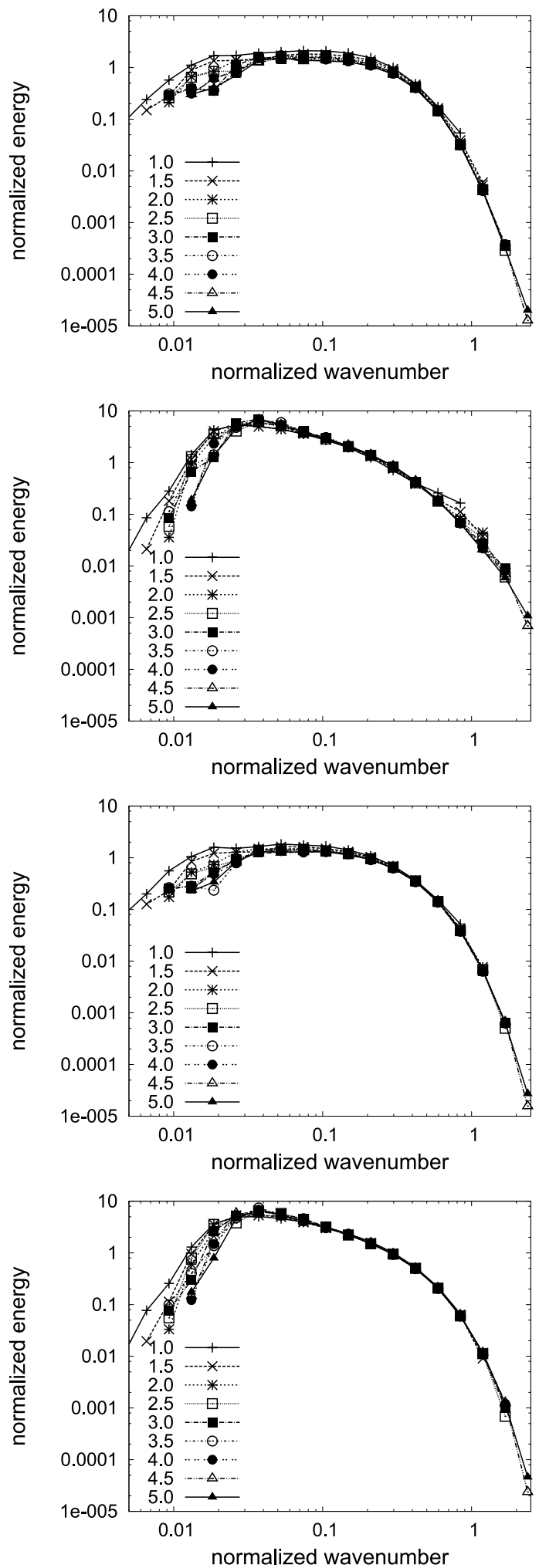

Fig. 3 Time series of the normalized kinetic and magnetic energy spectra; from top to bottom: $E_{j}^{(u)}$ of HMHD simulation, $E_{j}^{(b)}$ of HMHD simulation, $E_{j}^{(u)}$ of MHD simulation, and $E_{j}^{(b)}$ of MHD simulation, where the superscripts $u$ and $b$ denote kinetic and magnetic energy, respectively.

fluid advection and the Hall term, which rearrange the kinetic and magnetic energy distribution, vary more slowly than those induced by their mutual interactions. 

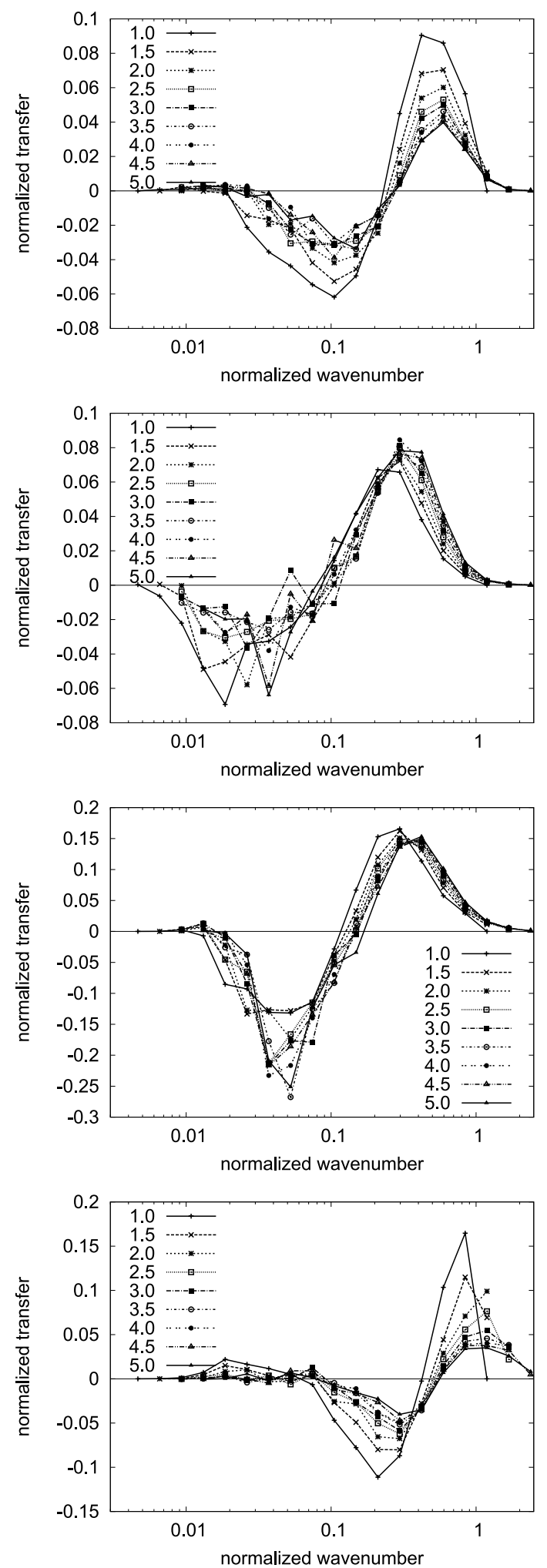

Fig. 4 Time series of normalized energy transfer in the HMHD simulation; from top to bottom: advection $\left\langle\boldsymbol{u}_{j}|\boldsymbol{u}| \boldsymbol{u}\right\rangle_{a d v}$, Lorentz force $\left\langle\boldsymbol{u}_{j}|\boldsymbol{b}| \boldsymbol{b}\right\rangle_{L o r}$, magnetic induction $\left\langle\boldsymbol{b}_{j}|\boldsymbol{b}| \boldsymbol{u}\right\rangle_{\text {Ind }}$, and the Hall term effect $\left\langle\boldsymbol{b}_{j}|\boldsymbol{b}| \boldsymbol{b}\right\rangle_{\text {Hall }}$.

An interesting feature of these systems is that although the energy transfers induced by the Lorentz force effect and the Hall term both involve the $\boldsymbol{j} \times \boldsymbol{b}$ field in their integrands,
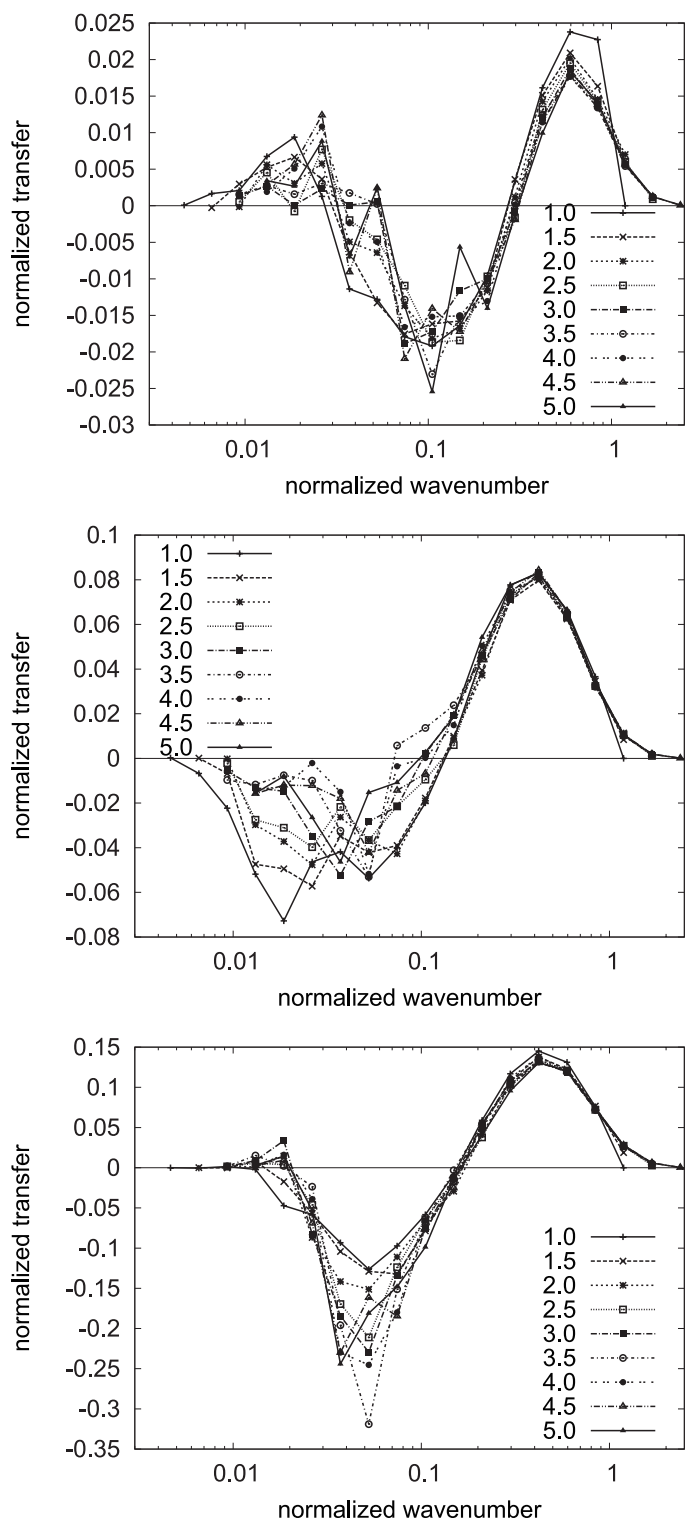

Fig. 5 Time series of normalized energy flux in the MHD simulation: from top to bottom: advection $\left\langle\boldsymbol{u}_{j}|\boldsymbol{u}| \boldsymbol{u}\right\rangle_{a d v}$, Lorentz force $\left\langle\boldsymbol{u}_{j}|\boldsymbol{b}| \boldsymbol{b}\right\rangle_{L o r}$, and magnetic induction $\left\langle\boldsymbol{b}_{j}|\boldsymbol{b}| \boldsymbol{u}\right\rangle_{\text {Ind }}$.

they converge to the stationary state at very different time scales.

In the MHD medium, energy transfer induced by the Lorentz force and magnetic induction is rapid and more effective than in the HMHD medium. The amplitude of the energy transfer due to fluid advection converges slightly more slowly than that of the other transfers. However, significant reduction in energy transfer, highly obvious in the HMHD medium, is not observed in the MHD medium.

\section{Discussion}

We compared the time series of the Fourier band-pass filtered spectra of the energies and the quadratic terms in the energy transfers in simulated MHD and HMHD systems. Although the normalized energy spectra converge 
to particular profiles, the amplitude of the energy transfers changes in time. The transfers related to mode interactions between the magnetic and velocity fields converges relatively quickly over dissipation range scales, while the amplitudes of nonlinear terms (fluid advection and the Hall term effect) relax relatively slowly. The relaxation times of both terms are prolonged in HMHD medium, suggesting that slow dynamical modes exist in the decay process.

This slow relaxation following convergence of the energy spectra seems to be related to the so-called "depression of nonlinearity" in fully developed turbulence. This phenomenon has aroused great interest among turbulence researchers and has been investigated in both neutral fluids (e.g. Refs.[7] and [8]) and magnetohydrodynamic (MHD) fluids [9]. The authors of these studies compared turbulent fields with phase randomized fields. In the present study, we found selective depression of triad mode interactions in HMHD turbulence by directly comparing the normalized triad interactions.

Since the amplitudes of the magnetic and velocity fields are normalized, the datasets collected at different times differ principally in the topological features of their vector fields. At present, we are investigating the physical reasons for this novel selective reduction phenomenon.
The authors express appreciation to the anonymous referee for the kind and valuable comments. This study was conducted under the auspices of the NIFS Collaboration Research Program (NIFS11KNSS016, NIFS10KNSS011) and KAKENHI (Grant-in-Aid for Scientific Research(C)) 22540509, 23540583.

[1] S.M. Mahajan and Z. Yoshida, Phys. Plasmas 7, 635 (2000).

[2] H. Miura and D. Hori, J. Plasma Fusion Res. Series 8, 73 (2009).

[3] K. Araki and H. Miura, Plasma Fusion Res. 5, S2048 (2010).

[4] K. Araki and H. Miura, Plasma Fusion Res. 6, 2401132 (2010).

[5] C.K. Chui, An introduction to wavelets (Academic Press, London, 1992) Chapt.1.

[6] K. Araki and H. Miura, J. Plasma Fusion Res. Series 9, 446 (2010).

[7] R.H. Kraichnan and R. Panda, Phys. Fluids 31, 2395 (1988).

[8] A. Tsinober, M. Ortenberg and L. Shtilman, Phys. Fluids 11, 2291 (1999).

[9] S. Servidio, W.H. Matthaeus and P. Dmitruk, Phys. Rev. Lett. 100, 095005 (2008). 